\title{
Personalizar un entorno para listas de ejercicios con el paquete answers
}

\author{
Walter Mora F. \\ wmora2@itcr.ac.cr \\ Escuela de Matemática \\ Instituto Tecnológico de Costa Rica
}

\begin{abstract}
Resumen. En este artículo se hace la implementación de varios entornos personalizados para hacer listas de ejercicios (y sus soluciones) con el paquete "answers" de LaTeX. El propósito que se persigue es hacer que el uso de este paquete sea un poco más amigable en la edición de libros y de material didáctico en general.
\end{abstract}

Palabras clave: LaTeX, paquete answers, listas de ejercicios en Latex.

Abstract. In this article several personalized environments are implemented to make lists of exercises (and corresponding solutions) using the LaTeX "answers" package. The purpose is to ease the use of this package for book and general didactic material edition.

KeyWords: LaTeX, answers package, LaTex exercise lists

\subsection{Introducción}

El paquete answers permite hacer listas de ejercicios en documentos $\mathrm{ET}_{\mathrm{E}} \mathrm{X}$. Usualmente solo hay que definir los entornos para cada ejercicio y para las soluciones, si hubiera.

Aunque el código de los ejemplos que siguen parece algo denso, eso no es problema porque ese código uno lo puede separar y "esconder" en un archivo de estilo o un archivo de "comandos" personales que 
2 Revista digital Matemática, Educación e Internet (http:\%\%www.tec-digital.itcr.ac.cr\%revistamatematica\%). Vol 14, No 1. Setiembre - Febrero 2014.

se carga al iniciar el documento.

Por ejemplo, en un formato de libro de la revista digital Matemática, Educación e Internet ((http:/ / www.tecdigital.itcr.ac.cr/revistamatematica/)) que usa esta personalización para las listas de ejercicios, el código está en el pquete de estilo. El documento inicia así,

Descargar plantilla del libro

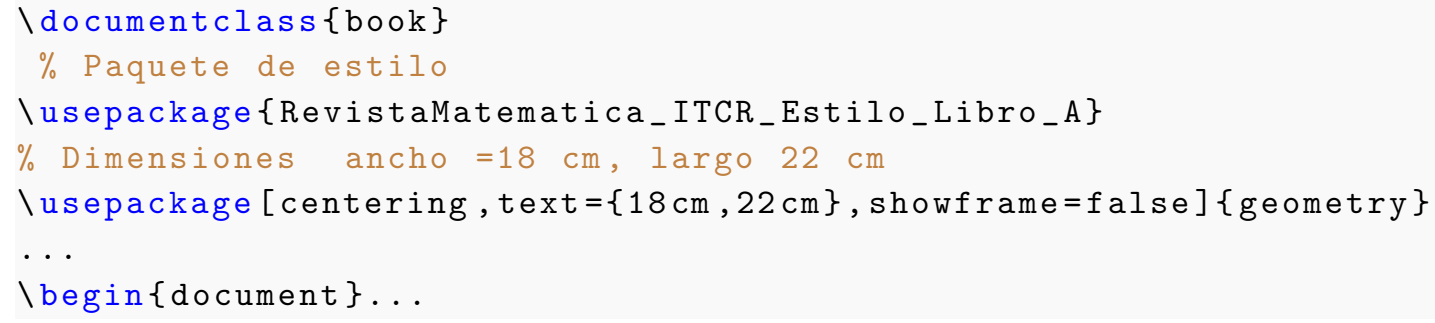

Ejemplo de un entorno. Un entorno mínimo para hacer listas de ejercicios con el paquete answers sería algo como

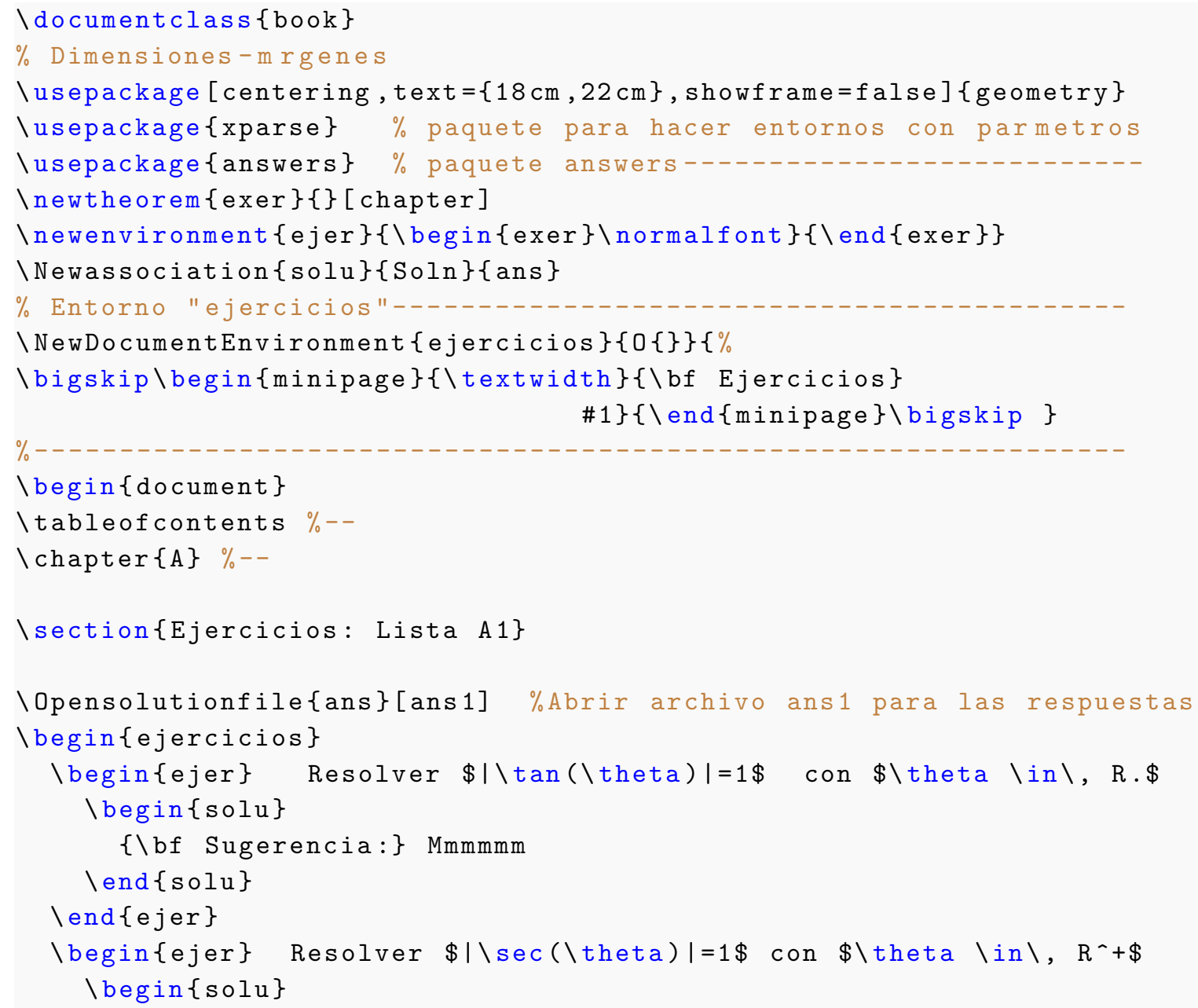




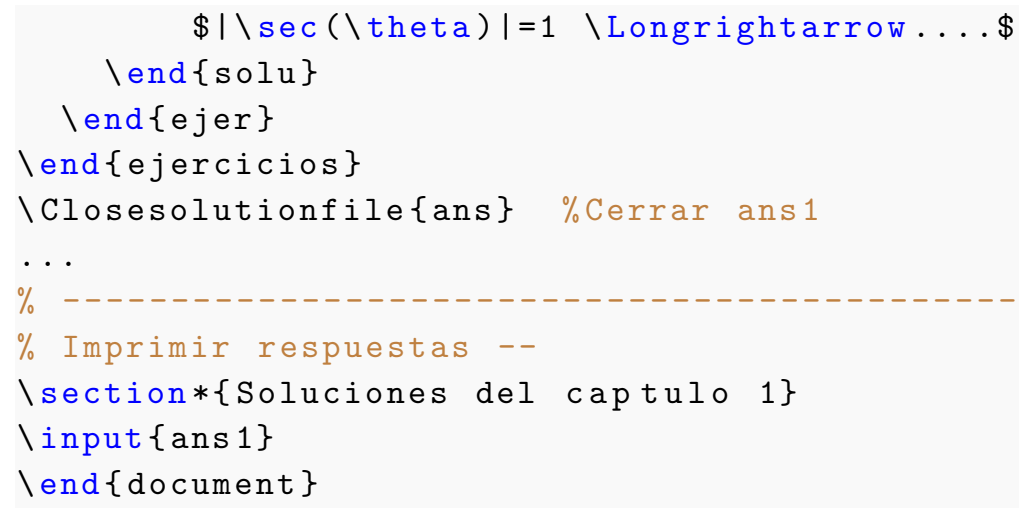

Como se ve, hay que tener control sobre la lista actual ans1, ans2, . . de ejercicios. Y esto en un libro un poco grande no deja de ser incómodo.

\subsection{Un entorno personalizado}

Para no tener que estar llevando un control de la lista actual de ejercicios ans1, ans2, ..., la idea es solo abrir un archivo "\ans \thechapter" por capítulo y cerrarlo al final de cada capítulo. El efecto es que todas las listas de ejercicios del capítulo $i$ se acumulan en el archivo "ansi", con su respectiva numeración. Así, al final del documento solo hay que abrir el archivo correspondiente a cada capítulo que tenga listas de ejercicios.

Una idea: Para abrir un archivo "ansj" para cada capítulo $j$ de manera automática, una idea es redefinir el entorno chapter de LTEX. Para hacer esto se usa \renewcommand y eso permite agregar los comandos para "cerrar la lista anterior de ejercicios si hubiera, y abrir una nueva lista para este capítulo" y no cambiar nada más del entorno chapter. Si el capítulo no tiene listas de ejercicios, solo se genera un archivo "ans" vacío. También sería bueno hacer esto sin que interfiera con el estilo del paquete answers de abrir y cerrar los archivos de respuestas.

Ajustes necesarios. Con la idea anterior, se necesita un contador de capítulos "\ansj". Este contador aumenta en uno cada vez que se hace una llamada \chapter. El comando \tableof contents hace una llamada a \chapter, así que si nuestro libro viene con una tabla de contenidos ( $\backslash$ tableofcontents), "\ansj" se debe inicializar en 0, así que nos sirve \ansj=\thechapter.

Así mismo, un prólogo o un prefacio que use \chapter*\{\} también aumentaría el contador. En este caso, poner \chapter $*\{\}$ en un entorno $\backslash$ begin $\{$ prologo $\ldots$. . \end } \{ \text { prologo\} resuelve el problema. Pero } en general, hay que tomar el control del libro para controlar los contadores.

Restricciones. Para no contaminar el código, podemos suponer hay un par de restricciones: El libro tiene un prólogo en un entorno (no aumentael contador) y tiene una tabla de contenidos, antes del primer capítulo.

En otro caso, solo habría que poner $\backslash a n s j=1$ antes del primer capítulo del libro. 
Modificar \chapter. La definición de chapter en el archivo book.cls es

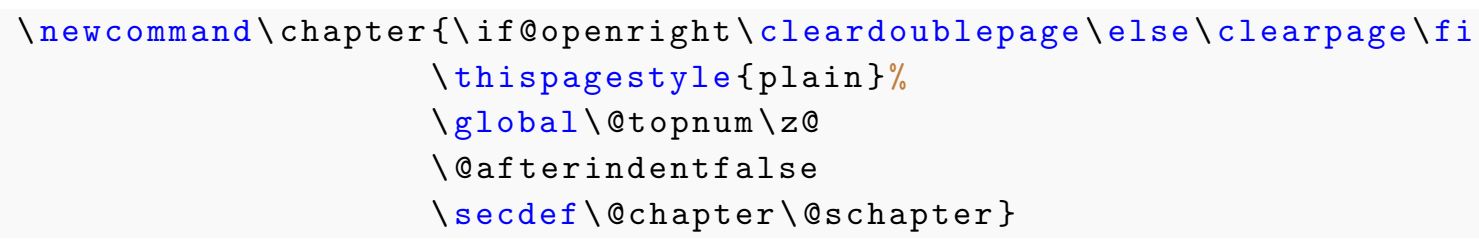

Para redefinir este comando usamos \let \stdchapter \chapter para guardar una copia de la definición original del comando \chapter como \stdchapter y usamos \stdchapter precisamente para devolvernos al comportamiento original de \chapter mientras infiltramos las líneas de código que nos interesa.

Solo hay que tener el cuidado de redefinir los dos sabores de \chapter, a saber \chapter [] \{\} y Ichapter $*\{\}$. Esto se hace, por supuesto, con \stdchapter [] \{\} y \stdchapter $*\{\}$

Cuando vamos a abrir y cerrar archivos ans j debemos usar \csname Opensolutionfile \endcsname para verificar si el comando ha sido definido. Igual para Closesolutionfile.

Para implementar la idea anterior, se agrega en el preámbulo del documento el código

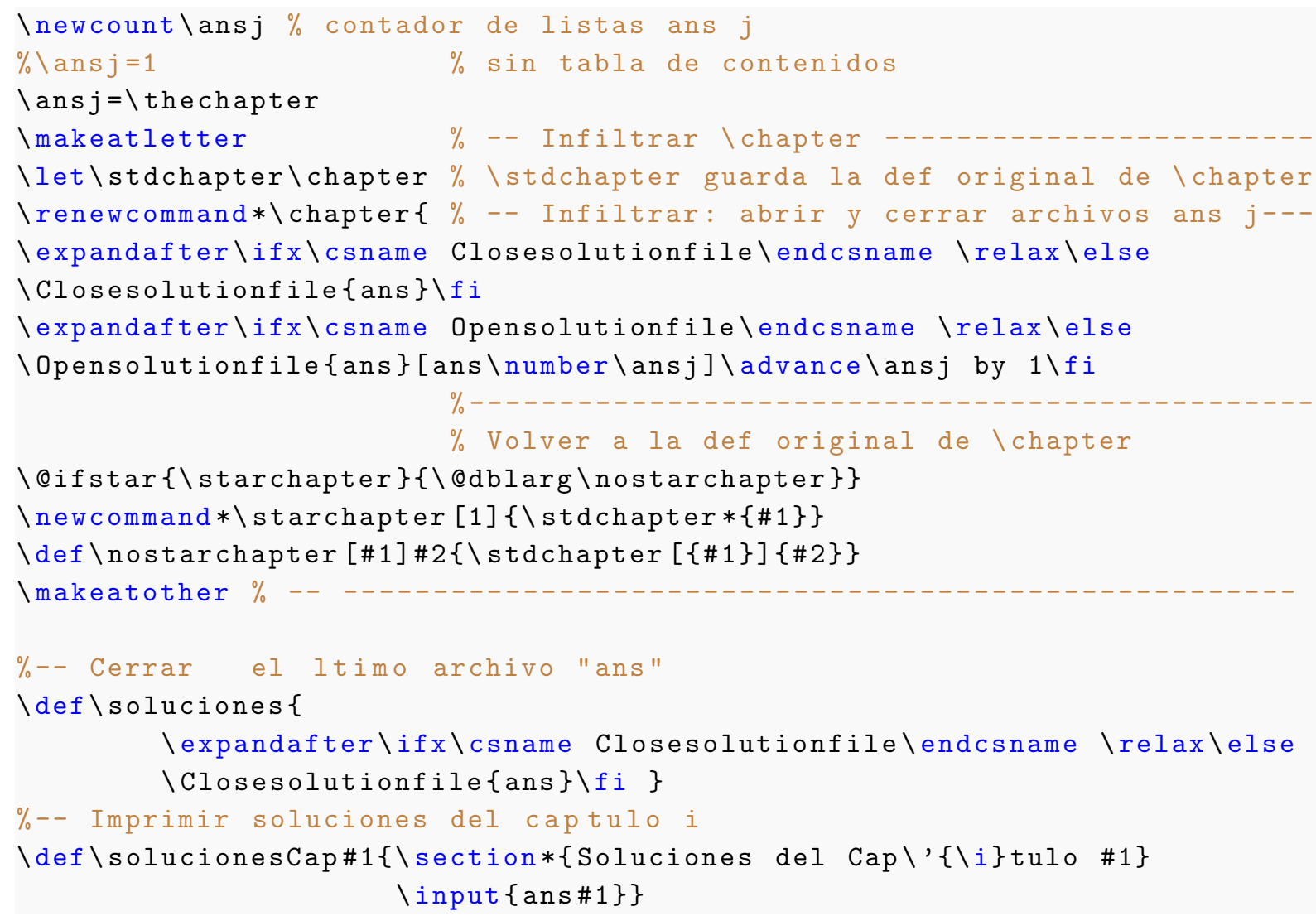


A partir de aquí ya podemos agregar listas de ejercicios (una o varias) en cada capítulo sin tener que abrir ni cerrar los archivos ans i. Ahora esto es automático. El código de un archivo de prueba sería,

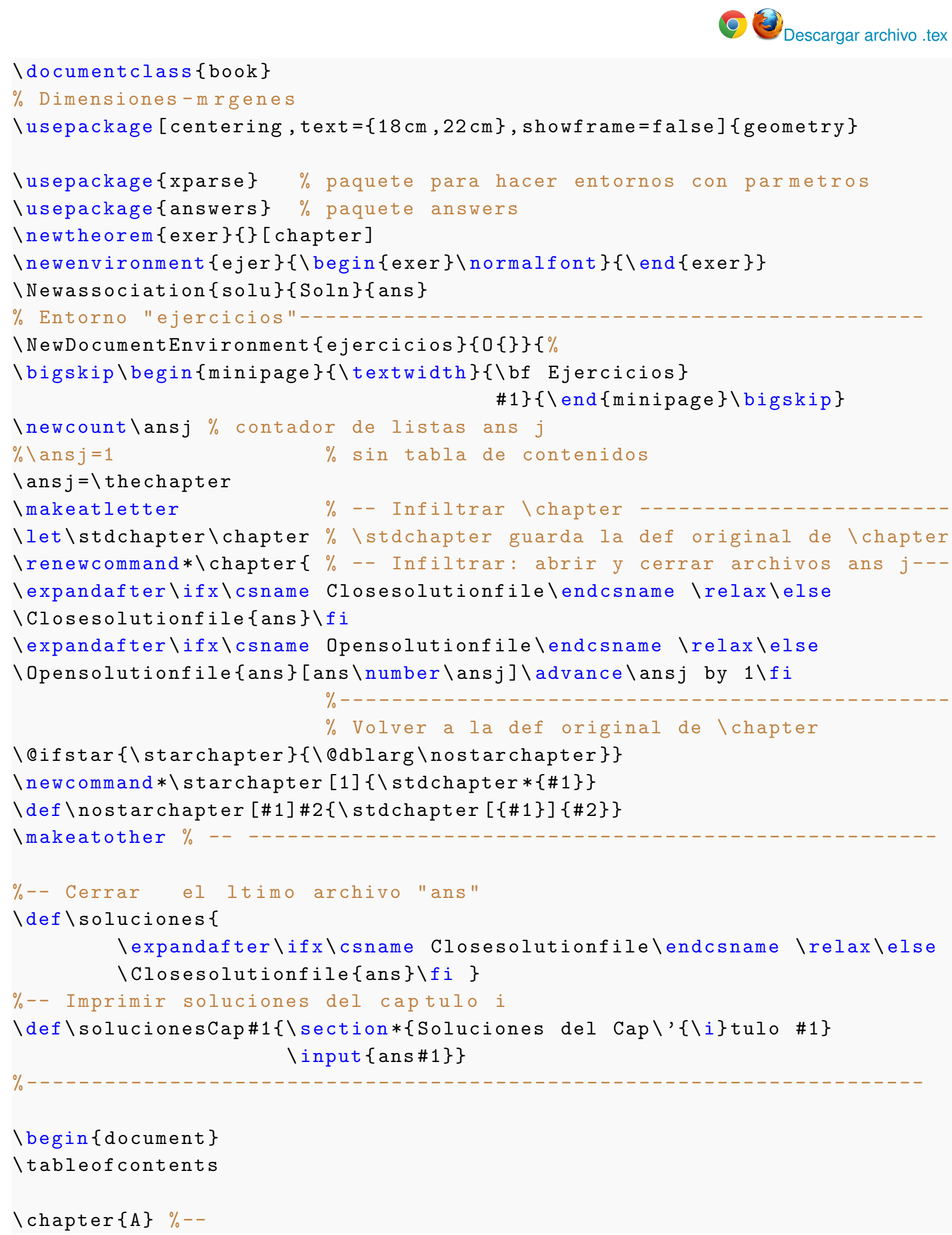




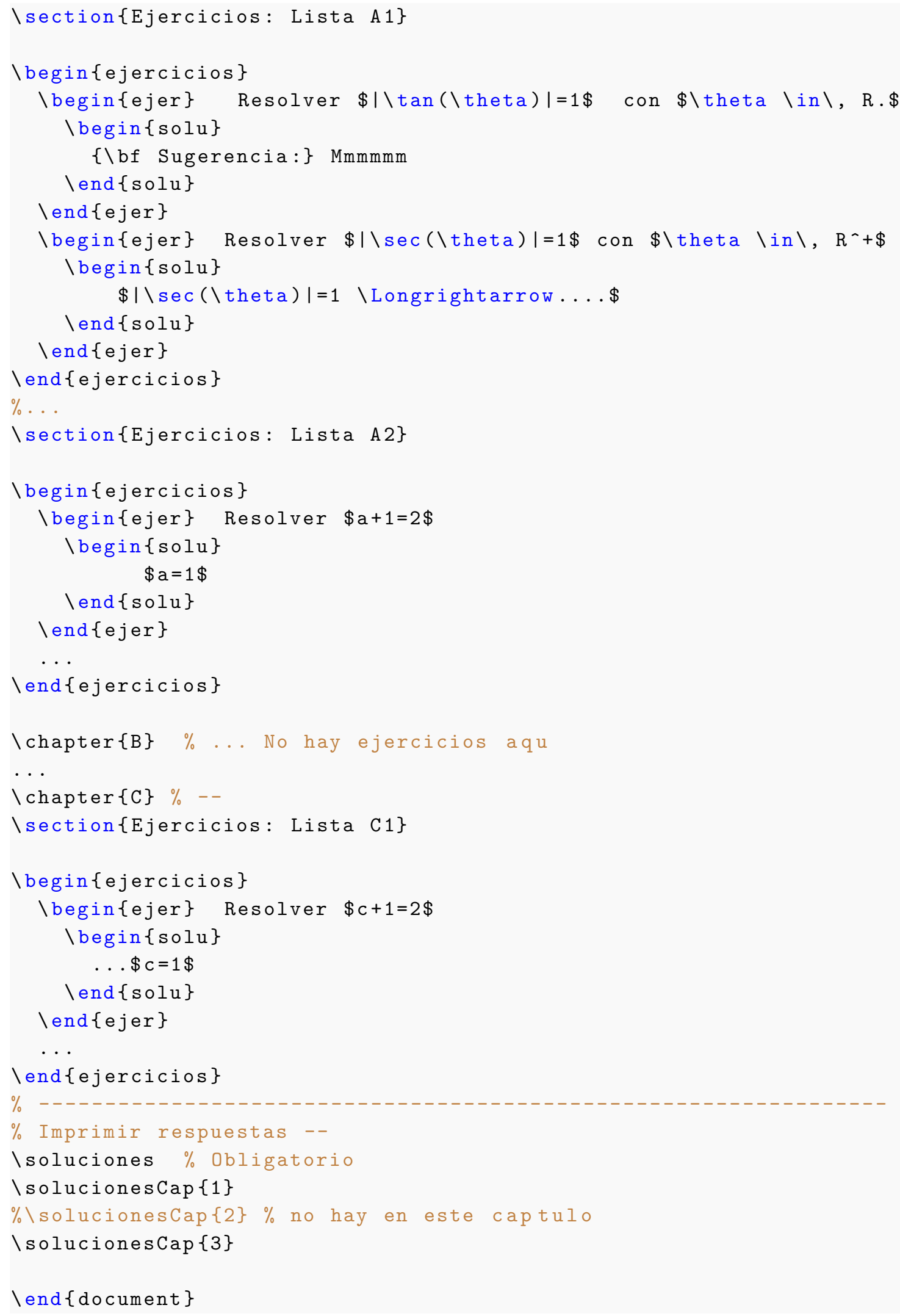




\subsection{Cajas para las listas de ejercicios}

Podemos crear un entorno de cajas para las listas de ejercicios, tal como

\section{Ejercicios}

1.1 Resolver $|\tan (\theta)|=1 \operatorname{con} \theta \in R$.

1.2 Resolver $|\sec (\theta)|=1 \operatorname{con} \theta \in R^{+}$

Para hacer esto, creamos una caja de fondo gris con un "nodo" rectangular en verde, donde va el título "Ejercicios". Todo esto se puede hacer con el paquete tcolorbox. Adicionalmente, la caja se debe declarar "breakable" para que se extienda a la siguiente página en caso de que sea necesario. Solo debemos agregar en el código del ejemplo anterior,

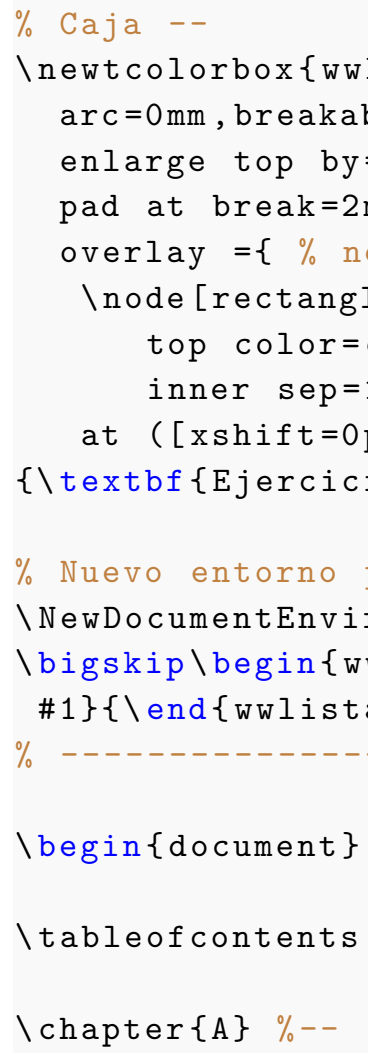




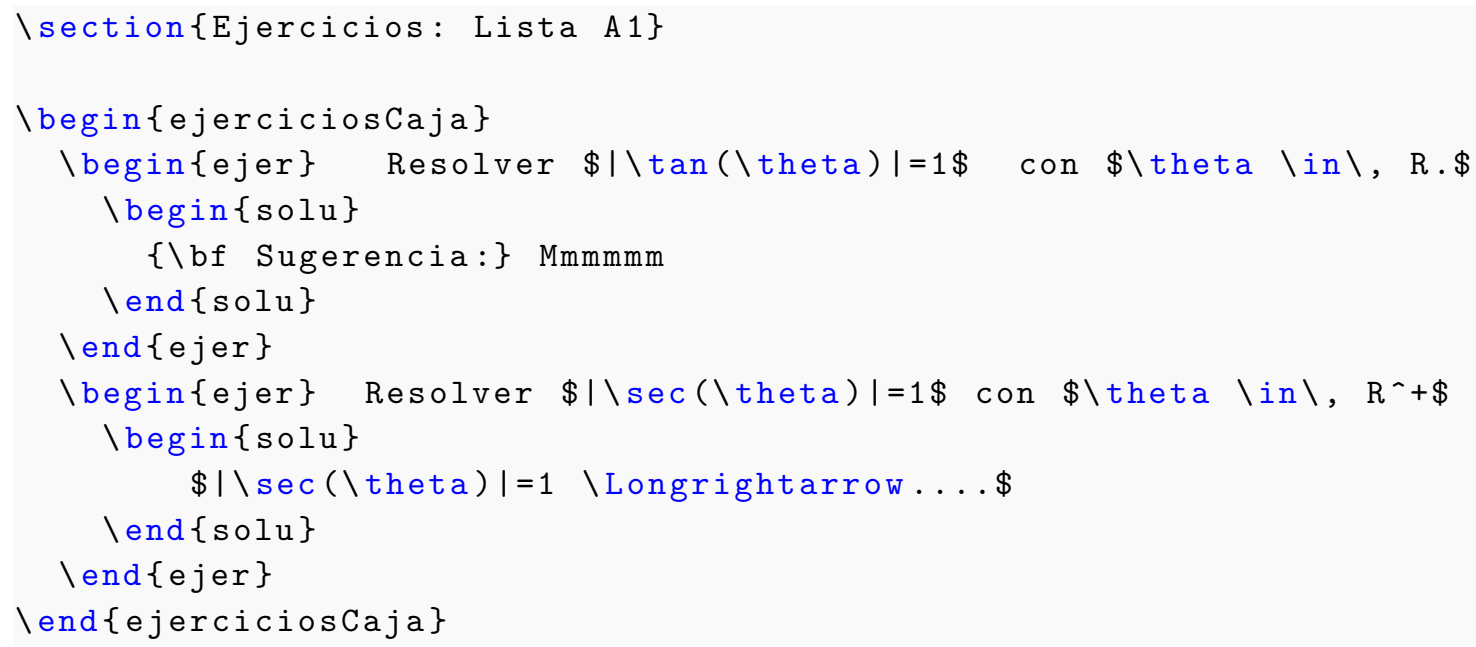

Código "encapsulado". Como deciamos, todo el código se puede "ocular" en un archivo de estilo o en un archivo .tex que se importa al principio del documento. Una plantilla de ejemplo se puede descargar aquí (9) Descargar plantilla del libro

\section{Bibliografía}

[1] Amy Hendrickson. "The Wonders of \csname... \endcsname". TUGboat, Volume 0 (2012), No. 0 Proceedings of the 2012 Annual Meeting. http://www.tug.org/tug2012

[2] "Custom Ichapter definition". http://tex.stackexchange.com/questions/60505/customchapter-definition.

[3] W. Mora, A. Borbón. "Edición de textos científicos con LaTeX.Composición, diseño editorial, Gráficos y Beamer." http://www.tec-digital.itcr.ac.cr/revistamatematica/Libros/LATEX/LaTeX_2013. pdf

[4] Thomas F. Sturm. "The LaTeX package tcolorbox". version 2.50. http://www.ctan.org/texarchive/macros/latex/contrib/tcolorbox

[5] Donald E. Knuth. "The TeXbook". Addison-Wesley, 1984.

[6] Norman Walsh. "Making TeX Work". O'Reilly \& Associates, Inc. http://makingtexwork. sourceforge.net/mtw/

[7] Victor Eijkhout "TeX by Topic, A TeXnician's Reference". Addison-Wesley Publishing Company. 